\title{
CONTINUITY OF MEASURABLE CONVEX AND BICONVEX OPERATORS
}

\author{
LIONEL THIBAULT
}

\begin{abstract}
We prove that a mapping from the product of two complete metrizable vector spaces into a topological vector space which is separately universally measurable and separately convex with respect to a convex cone is continuous.
\end{abstract}

0. Introduction. If $X$ is a complete separable vector space and if $f$ is a midpoint convex mapping from $X$ into the real line $\mathbf{R}$ which is Christensen measurable, then $\mathrm{F}$. Fischer and Z. Slodkowski have proved in [4] that $f$ is continuous by showing that $\{(x, r) \in X \times \mathbf{R}: f(x)<r\}$ is open in $X \times \mathbf{R}$. Results of this kind about continuity of universally measurable morphisms were obtained before by $\mathrm{A}$. Douady and $\mathrm{L}$. Schwartz (see [7]) for linear operators between locally convex spaces, and by J. P. R. Christensen in [3] for homomorphisms between topological complete metrizable groups.

In this paper we are concerned with the study of convex operators taking values in a vector space. Vector analogues of the preceding results are proved. In fact, we establish that a separately universally measurable mapping $f: X \times Y \rightarrow F$, where $X$ and $Y$ are two complete metrizable vector spaces and $F$ is any topological vector space, is continuous whenever it is separately midpoint convex with respect to a convex cone.

Let us also note that many other interesting results about continuity of convex operators are given by J. M. Borwein in [1].

1. Preliminaries. Throughout this paper $F$ will denote a (real separated) topological vector space and $X$ and $Y$ two real complete metrizable topological vector spaces.

Let $P$ be a convex cone in $F$, i.e. $t P+s P \subset P$ for all $t, s \geqslant 0$. One says that $P$ is normal if there is a base of neighbourhoods $V$ of zero with

$$
V=(V+P) \cap(V-P) \text {. }
$$

Such neighbourhoods are said to be full. Many properties and examples of normal convex cones can be found in [7]. In the sequel we shall always assume that $P$ is a normal convex cone in $F$.

A subset $B$ of a topological space $S$ is universally measurable if for each finite measure $m$ over the Borel tribe $\mathscr{B}(S)$ the set $B$ belongs to the $m$-completion of $\mathscr{B}(S)$. The set of universally measurable subsets of $S$ will be denoted by $थ(S)$.

Received by the editors May 15, 1983.

1980 Mathematics Subject Classification. Primary 46A40, 46A55.

Key words and phrases. Convex operators, biconvex operators, universally measurable mappings. 
A mapping $f: S \rightarrow F$ is universally measurable if for each open subset $\Omega$ of $F$ the set $f^{-1}(\Omega)$ is in $\mathcal{U}(S)$.

1.1. Remark. As a direct consequence of Proposition 8 in [5, p. 12], for each continuous mapping $f$ from a topological space $S$ into a topological space $T$, one has $f^{-1}(B) \in \mathcal{U}(S)$ for each $B \in \mathcal{U}(T)$.

Let us recall the following consequence of a very nice and important result of Christensen.

1.2. Proposition. If $X=\cup_{n \in \mathrm{N}} B_{n}$ is a countable union of universally measurable subsets, then there exists an integer $k$ such that $B_{k}-B_{k}=\left\{x-y: x, y \in B_{k}\right\}$ is a neighbourhood of zero.

Proof. This is a direct consequence of Theorem 7.1 in [3].

\section{Midpoint convex operators.}

2.1. Definition. One says that $f: X \rightarrow F$ is $P$-convex if

$$
f(t x+(1-t) y) \in t f(x)+(1-t) f(y)-P \text { for all } t \in[0,1],
$$

and $f$ is midpoint $P$-convex if (2.1) holds for $t=1 / 2$.

One observes that additive mappings are always midpoint convex.

REMARK. It is easily shown that $f$ is midpoint $P$-convex if and only if

$$
f\left(2^{-n} k x+\left(1-2^{-n} k\right) y\right) \in 2^{-n} k f(x)+\left(1-2^{-n} k\right) f(y)-P
$$

for all $x, y \in X, k$ and $n \in \mathbf{N}$ with $0 \leqslant k \leqslant 2^{n}$. It follows that a midpoint $P$ convex operator with closed epigraph is convex.

2.2. Lemma. A midpoint P-convex operator $f: X \rightarrow F$ is continuous at $a \in X$ if and only if $f$ is upper semicontinuous at $a$ in the following sense: for every neighbourhood $W$ of zero in $F$ there exists a neighbourhood $V$ of zero in $X$ such that

$$
f(a+x) \in f(a)+W-P \text { for each } x \in V .
$$

Proof. It is clearly enough to show that the condition is sufficient. Let $W$ be any neighbourhood of zero in $F$. Choose a full circled neighbourhood $W_{0}$ of zero in $Y$ with $W_{0} \subset W$ and a circled neighbourhood $V$ of zero in $X$ satisfying

$$
f(a+x)-f(a) \in W_{0}-P \text { for all } x \in V .
$$

As $f$ is midpoint $P$-convex, we have for each $x \in V$

and hence

$$
f(a) \in \frac{1}{2} f(a-x)+\frac{1}{2} f(a+x)-P
$$

$$
f(a+x)-f(a) \in f(a)-f(a-x)+P \subset W_{0}+P+P=W_{0}+P .
$$

Making use of relation (2.3) once again we obtain

$$
f(a+x)-f(a) \in\left(W_{0}-P\right) \cap\left(W_{0}+P\right)=W_{0} \subset W
$$

for each $x \in V$.

2.3. Proposition. Let $f: X \rightarrow F$ be a universally measurable midpoint P-convex operator; then:

(i) $f$ is continuous,

(ii) if $P$ is closed, $f$ is a continuous $P$-convex operator. 
Proof. Let us begin by proving the continuity. Let $a$ be any point in $X$. Put $g(x)=f(a+x)-f(a)$. The operator $g$ is obviously a midpoint convex operator with $g(0)=0$ and by Remark 1.1 it is universally measurable. Let $W_{0}$ be a neighbourhood of zero in $F$. Choose an open circled neighbourhood $W$ of zero with $W+W \subset W_{0}$. For each nonnegative integer $n$ consider the universally measurable set

$$
B_{n}=\left\{x \in X: g(x) \in 2^{n+1} W, g(-x) \in 2^{n+1} W\right\} .
$$

Since $X=\cup_{n \in \mathrm{N}} B_{n}$, there exists, by Proposition 1.2, an integer $k$ such that $B_{k}-B_{k}$ is a neighbourhood of zero in $X$, and for each $x$ in the neighbourhood of zero $V:=$ $\frac{1}{2} B_{k}-\frac{1}{2} B_{k}=\frac{1}{2} B_{k}+\frac{1}{2} B_{k}$ we may write $x=\frac{1}{2} b+\frac{1}{2} b^{\prime}$ with $b, b^{\prime} \in B_{k}$,

$$
g(x) \in \frac{1}{2} g(b)+\frac{1}{2} g\left(b^{\prime}\right)-P \subset 2{ }^{k} W+2{ }^{k} W-P \subset 2{ }^{k} W_{0}-P
$$

and hence

$$
2^{-k} g(x) \in W_{0}-P \text {. }
$$

As $g$ is midpoint $P$-convex with $g(0)=0$, we have for each $x \in V$,

$$
g\left(2^{-k} x\right) \in 2^{-k} g(x)-P \subset W_{0}-P
$$

and hence by Lemma $2.2, g$ is continuous at 0 , which implies that $f$ is continuous.

If $P$ is closed, then the continuity of $f$ and relation (2.2) easily imply that $f$ is $P$-convex.

\section{Midpoint biconvex operators.}

3.1. Definition. A mapping $f: X \times Y \rightarrow F$ is called a midpoint P-biconvex operator if for each $(x, y) \in X \times Y$ the mappings $f(x, \cdot)$ and $f(\cdot, y)$ are midpoint $P$-convex operators.

3.2. Proposition. Let $f: X \times Y \rightarrow F$ be a separately universally measurable midpoint $P$-biconvex operator; then:

(i) $f$ is continuous,

(ii) if $P$ is closed, $f$ is a continuous $P$-biconvex operator.

Proof. Let $(c, d)$ be any point in $X \times Y$. Put $g(x, y)=f(c+x, d+y)-$ $f(c, d+y)$. The mapping $g$ is separately universally measurable and $g(0,0)=0$. Let $\left(x_{n}, y_{n}\right)_{n \in \mathrm{N}}$ be any sequence in $X \times Y$ converging to zero and let $W_{0}$ be any full circled neighbourhood of zero in $F$. Choose an open neighbourhood $W$ of zero satisfying $W+W \subset W_{0}$. For each $x \in X$, by Proposition 2.3 , the mapping $g(x, \cdot)$ is continuous and hence the set $\left\{g\left(x, y_{n}\right): n \in \mathbf{N}\right\}$ is topologically bounded in $F$ as $\{0\} \cup\left\{y_{n}: n \in \mathbf{N}\right\}$ is compact in $Y$. So if we put, for each $p \in \mathbf{N}$,

$$
B_{p}=\left\{x \in X: g\left(x, y_{n}\right) \in 2^{p+1} W \text { and } g\left(-x, y_{n}\right) \in 2^{p+1} W, \forall n \in \mathbf{N}\right\},
$$

then $X=\cup_{p \in \mathbf{N}} B_{p}$ and hence, by Proposition 1.2, there exists an integer $k$ and a circled neighbourhood $V$ of zero in $X$ with $V \subset \frac{1}{2} B_{k}-\frac{1}{2} B_{k}=\frac{1}{2} B_{k}+\frac{1}{2} B_{k}$. Therefore, for each $x=\frac{1}{2} b+\frac{1}{2} b^{\prime} \in V$ with $b, b^{\prime} \in B_{k}$ and each $n \in \mathbf{N}$, invoking the midpoint convexity of $g\left(\cdot, y_{n}\right)$ we have

$$
g\left(x, y_{n}\right) \in \frac{1}{2} g\left(b, y_{n}\right)+\frac{1}{2} g\left(b^{\prime}, y_{n}\right)-P \subset 2^{k} W+2^{k} W-P \subset 2^{k} W_{0}-P
$$


and hence again, by the midpoint convexity of $g\left(\cdot, y_{n}\right)$ and the relation $g\left(0, y_{n}\right)=0$, we have

$$
g\left(2^{-k} V, y_{n}\right) \subset\left(W_{0}-P\right) \cap\left(W_{0}+P\right)=W_{0} \quad \text { for every } n \in \mathbf{N} .
$$

As $\lim _{n \rightarrow \infty} x_{n}=0$, we may conclude that $\lim _{n \rightarrow \infty} g\left(x_{n}, y_{n}\right)=0$ and the proof is complete since $y \rightarrow f(c, d+y)$ is continuous.

The above proof also gives the following result.

3.3. Proposition. Let $f: X \times Y \rightarrow F$ be a universally measurable midpoint $P$ convex-concave operator, that is $f(\cdot, y)$ and $-f(x, \cdot)$ are midpoint $P$-convex for each $(x, y) \in X \times Y$; then:

(i) $f$ is continuous,

(ii) if $P$ is closed, $f$ is a continuous $P$-convex-concave operator.

Remarks. (1) If $X$ and $Y$ are also separable and if $\mathcal{C}(X)$ denotes the tribe of Christensen measurable subsets of $X$, that is (see [4]) the set of subsets $C \subset X$ for which there exist two universally measurable subsets $A$ and $M$, a probability measure $m$ on $\mathscr{Q}(X)$ and a subset $N \subset M$ such that $C=A \cup N$ and $m(x+M)=0$ for all $x \in X$, the above result still holds whenever $f$ is separately $\mathcal{C}(X)$ and $\mathcal{C}(Y)$ measurable.

(2) Results in the line of Proposition 3.2 about equicontinuous families of biconvex or concave-convex operators can be found in [6].

\section{REFERENCES}

I. J. M. Borwein, Continuity and differentiability properties of convex operators, Proc. London Math. Soc. (3) 44 (1982), 420-444.

2. J. P. R. Christensen, Borel structures in groups and semigroups, Mat. Scand. 28 (1971), 124-128.

3. Topologv and Borel structure, North-Holland, Amsterdam and American Elsevier, New York, 1974.

4. P. Fischer and Z. Slodkowski, Christensen zero sets and measurable convex functions, Proc. Amer. Math. Soc. 79 (1980), 449-453.

5. J. Hoffmann-Jorgensen, The theory of analytic sets, Aarhus Universitet Danmark, various publication series, no. 10, 1970.

6. M. Jouak and L. Thibault, Equicontinuity of families of convex and concave-convex operators (to appear).

7. A. L. Peressini, Ordered topological vector spaces, Harper \& Row, New York, 1971.

8. A. L. Schwartz, Sur le théorème du graphe fermé, C. R. Acad. Sci. Paris Sér A 263 (1966), 602-605.

9. L. Thibault, Continuity of measurable convex multifunctions (to appear).

Departement de Mathematiques et d'Informatique, Université de Pau, 64000 Pau, France 